HELICOBACTER PYLORI

\title{
Transactivation of the epidermal growth factor receptor by cag+ Helicobacter pylori induces upregulation of the early growth response gene Egr-1 in gastric epithelial cells
}

\author{
S Keates, A C Keates, S Nath, R M Peek Jr, C P Kelly
}

See end of article for authors' affiliations

.....................

Correspondence to: Dr S Keates, Division of Gastroenterology, Dana 601 , Beth Israel Deaconess Medical Center, 330 Brookline Ave, Boston, MA 02215, USA; skeates@ bidmc.harvard.edu

Revised version received 11 April 2005

Accepted for publication 28 April 2005

Published online first

29 April 2005

\begin{abstract}
Background and aims: Helicobacter pylori, in particular cytotoxin associated gene (cag)+ strains, have been shown to enhance gastric epithelial cell proliferation in vivo, an effect that likely contributes to gastric carcinogenesis. Early growth response gene 1 (Egr-1) is a crucial regulator of cell growth, differentiation, and survival, which is known to play a role in carcinogenesis and cancer progression. The aims of this study were to: (1) examine whether $H$ pylori could upregulate Egr-1 in gastric epithelial cell lines; (2) determine whether there was a differential response to infection with different strains; (3) examine the role of the cag pathogenicity island in this process; and (4) elucidate the molecular mechanisms leading to Egr1 upregulation.

Methods and results: We found that infection of AGS cells with cag+ $H$ pylori resulted in a rapid (12 hours) but transient increase in Egr-1 mRNA and protein levels whereas coculture with cag - isolates did not elicit this response. Furthermore, two independent cagE- isogenic mutants of $H$ pylori also demonstrated impaired ability to upregulate Egr-1. Upregulation of Egr-1 protein was inhibited by the extracellular regulated kinase (ERK)1/2 inhibitor PD98059 and overexpression of dominant negative MEK1 downregulated Egr-1 luciferase reporter gene activity. Treatment of AGS cells with the epidermal growth factor receptor (EGFR) kinase inhibitors PD153035 and AG1478 resulted in a reduction in $H$ pylori mediated Egr-1 upregulation, demonstrating that EGFR transactivation plays a role in this early cellular process.

Conclusions: Our findings show that cag $+\mathrm{H}$ pylori cause rapid induction of Egr-1 in gastric epithelial cells which may contribute to $H$ pylori mediated pathogenesis.
\end{abstract}

$\mathrm{H}$ elicobacter pylori is a gastric pathogen that infects over half the world's population. This Gram negative bacterium colonises the epithelial layer of the stomach and induces a state of chronic inflammation that can lead to the development of life threatening diseases, including gastric and duodenal ulceration and distal gastric adenocarcinoma. ${ }^{1-3}$ The interaction between $H$ pylori and gastric epithelial cells results in activation of multiple signalling cascades such as mitogen activated protein kinase (MAP) pathways, ${ }^{4-6}$ and also activation of transcription factors such as nuclear factor $\kappa \mathrm{B}$ and $\mathrm{AP}-1$ which have been shown to be important in regulating the host inflammatory response..$^{7-9}$

$H$ pylori strains can be broadly divided into cytotoxin associated gene (cag) + strains and cag - strains, depending on whether or not they possess a $40 \mathrm{~kb}$ region of genes known as the cag pathogenicity island (cag PAI). ${ }^{10}$ The genes that comprise the cag PAI encode for a type IV secretion system which acts as a molecular syringe to deliver the immunodominant CagA protein into the cytosol of the host cell. ${ }^{11-14}$ In addition to this delivery of peptidoglycan to host cells by the bacterial type IV secretion has also been demonstrated. ${ }^{15} \mathrm{H}$ pylori strains that possess the cag PAI have been found to induce more severe gastritis and augment the risk of developing peptic ulcer disease and distal gastric cancer. ${ }^{14}$ Furthermore, it has been shown that $H$ pylori cag+ strains selectively enhance proliferation and attenuate apoptosis of epithelial cells in vivo compared with cag- strains. ${ }^{16}{ }^{17}$ Differences have also been observed in the abilities of cag+ and cag- $H$ pylori strains to activate various signalling cascades in infected gastric epithelial cell lines. ${ }^{48}$

Early growth response gene $\mathrm{l}$ (Egr-1) is a $80-82 \mathrm{kDa}$ transcription factor also known as zif268, NGFI-A, Krox24, and TIS8. It is rapidly and transiently induced by a number of extracellular stimuli, including growth factors, cytokines, and injury related stimuli. ${ }^{18}$ Egr-1 is functionally implicated in numerous critical biological processes, including inflammation, cell proliferation, cell differentiation, vascular wound response, and cancer progression. ${ }^{18}$ Interestingly, Egr- 1 levels have been found to be elevated in gastric cancer tissues and it has been suggested that Egr-1 may play an important role in carcinogenesis and cancer progression in the stomach. ${ }^{19}$

In this study, we set out to examine whether $H$ pylori could induce an increase in Egr-1 in gastric epithelial cells and the mechanism regulating this effect. We also examined whether there were differences between strains in their ability to upregulate Egr-1 and, in particular, to assess the importance of the cag PAI in this process.

\footnotetext{
Abbreviations: Egr-1, early growth response gene 1; EGFR, epidermal growth factor receptor; ERK, extracellular regulated kinases; MAP kinases, mitogen activated protein kinases; PAI, pathogenicity island; cag, cytotoxin associated genes; PMA, phorbol-12-myristate-13acetate; $\mathrm{MOI}$, multiplicity of infection; GAPDH, glyceraldehyde-3phosphate dehydrogenase; PCR, polymerase chain reaction; EMSA, electrophoretic mobility shift assay; JNK, c-Jun $\mathrm{N}$-terminal kinase
} 


\section{MATERIALS AND METHODS}

Cell culture and reagents

AGS gastric epithelial cells (American Type Culture Collection, Rockville, Maryland, USA) were maintained as previously described. ${ }^{7}$ Cell culture experiments were carried out using 6,12 , or 24 well or $100 \mathrm{~mm}$ polypropylene tissue culture plates (Corning Costar, Cambridge, Massachusetts, USA). All experiments were carried out using confluent monolayers unless otherwise stated. Phorbol-12-myristate13-acetate (PMA) and pharmacological inhibitors AG1478, SB203580, PD98059, PD153035, SP600125, and actinomycin D were all obtained from Calbiochem (La Jolla, California, USA).

\section{$H$ pylori strains, clinical isolates, and isogenic mutants}

$H$ pylori were cultured and prepared as previously described. ${ }^{20}$ Unless otherwise stated, experiments were performed using the cagA+/vacA+H pylori strain 43504 (American Type Culture Collection). Isogenic $H$ pylori mutants lacking the cagE or cag $A$ gene were studied together with their parental cag + toxigenic wild-type strain $60190 .{ }^{21}$ A second set of $H$ pylori isogenic mutants lacking either the cagE or cagA gene were also studied along with their parental cag+ toxigenic wild-type strain J166. Mutants and clinical isolates J44 (cag-, vacA sla $\mathrm{m} 2$ ), J238 (cag+, vacA sla m2), J68 (cag-, vacA s2 m2), 107A (cag-, vacA s2 $\mathrm{m} 2$ ), and $\mathrm{J} 166$ (cagt, vacA slb ml) were obtained from Vanderbilt University Campylobacter and Helicobacter Laboratory (Nashville, Tennessee, USA). All clinical isolates were obtained from patients with gastritis except for J166 which was from a patient with duodenal ulcer disease.

\section{Western blotting}

AGS cells were grown on 12 well plates and maintained in serum free medium for 24 hours prior to the experiment to avoid cell stimulation by serum growth factors. $H$ pylori were added in serum free medium and cocultured for varying lengths of time (multiplicity of infection (MOI) 20:1; bacteria:cell). At the end of the experiment, monolayers were washed three times with phosphate buffered saline and lysed. Samples were loaded onto an $8 \%$ sodium dodecyl sulphate-polyacrylamide gel and transferred to nitrocellulose membranes (Bio-rad, Hercules, California, USA). Western blotting analysis was carried out using Egr-l and extracellular regulated kinase (ERK) 2 antibodies from Santa Cruz Biotechnology (California, USA), an actin antibody from Sigma-Aldrich (St Louis, Missouri, USA), and a phosphospecific-ERKl/2 (Cell Signaling Biotechnology, Beverly, Massachusetts, USA).

\section{Analysis of Egr-1 and GAPDH mRNA levels using real time reverse transcription-PCR (Taqman assay)}

Total RNA was isolated from AGS cells using guanidine isothiocyanate-phenol-chloroform extraction. RNA ( $1 \mu \mathrm{g})$ was reversed transcribed using random hexamer primers and Moloney murine leukaemia virus transcriptase, as previously described, ${ }^{22}$ and the resulting cDNA was stored at $-80^{\circ} \mathrm{C}$.

Egr-1 and glyceraldehyde-3-phosphate dehydrogenase (GAPDH) mRNA levels were determined using real time polymerase chain reaction (PCR) with a GeneAmp 5700 sequence detection system (ABI/Perkin-Elmer). cDNA was incubated for two minutes at $50^{\circ} \mathrm{C}$, denatured for 10 minutes at $95^{\circ} \mathrm{C}$, and then subjected to 40 cycles of annealing at $55^{\circ} \mathrm{C}$ for 20 seconds, extension at $60^{\circ} \mathrm{C}$ for one minute, followed by denaturation at $95^{\circ} \mathrm{C}$ for 15 seconds. The gene specific primers used were Egr-1 sense primer, 5' CCC GTT CGG ATC CTT TCC T 3'; Egr-1 antisense primer, 5' CAG CAT CAT
CTC CTC CAG CTT 3'; GAPDH sense primer, 5' GAC CAC AGT CCA TGC CAT CA 3'; and GAPDH antisense primer, 5' CAT CAC GCC ACA GTT TCC C 3'. To detect amplicons generated using the gene specific primers, dual labelled fluorogenic (Taqman) probes containing FAM (at the $5^{\prime}$ end) and TAMRA (at the 3' end) were synthesised (Sigma-Genosys, The Woodlands, Texas, USA). The Taqman probes used were: Egr-1, 5' ACT CGC CCA CCA TGG ACA ACT ACC C $3^{\prime}$ and GAPDH, 5' ACC CAG AAG ACT GTG GAT GGC CCC 3'. Egr- 1 levels in each sample were normalised to GAPDH expression, and the relative change in mRNA level was expressed as fold induction compared with untreated cells using the $-\Delta \Delta \mathrm{CT}$ method.

\section{Electrophoretic mobility shift assay (EMSA)}

Confluent $100 \mathrm{~mm}$ plates of AGS cells were exposed to $H$ pylori over a four hour time course and nuclear extracts prepared as previously described. ${ }^{7}$ Double stranded oligonucleotides containing consensus binding sites for Egr were obtained commercially (Santa Cruz Biotechnology). The oligonucleotides were end labelled using T4 polynucleotide kinase in the presence of $\left[\alpha-{ }^{32} \mathrm{P}\right]$ ATP and then purified on a Sephadex G-25 spin column (Boehringer Mannheim, Indianapolis, Indiana, USA). Binding reactions $(20 \mu \mathrm{l})$ contained $0.1 \mathrm{ng}(\sim 15000 \mathrm{cpm})$ of double stranded probe, 5-12 $\mu \mathrm{g}$ of extracted protein, $2 \mu \mathrm{g}$ of poly(dI-dC) (Pharmacia, Piscataway, New Jersey, USA), $10 \mathrm{mmol} / \mathrm{l} 2-\mathrm{ME}$, and $1 \%$ Ficoll. After first incubating the protein extracts for $10 \mathrm{~min}$ utes at room temperature, the radiolabelled probe was added. After an additional 30 minutes at room temperature, reaction mixtures were then loaded onto a non-denaturing $4.5 \%$ polyacrylamide gel in $0.2 \mathrm{~mol} / \mathrm{l}$ glycine, $25 \mathrm{mmol} / \mathrm{l}$ Tris $\mathrm{HCl}$, and $1 \mathrm{mmol} / \mathrm{l}$ EDTA. The gel was run, dried, and exposed to autoradiography film for $6-18$ hours at $--80^{\circ} \mathrm{C}$ with an intensifying screen. Supershift analyses were performed with anti-Egr-l and anti Sp-1 (Santa Cruz Biotechnology) to confirm the identity of the bound protein. Antibodies were added to the DNA probe at the start of the 30 minute incubation. In some experiments, binding specificity was also determined by competition with excess unlabelled probe.

\section{Transient transfection of AGS cells with an Egr-1 luciferase reporter}

AGS cells were transfected using Transfast (Promega, Madison, Wisconsin, USA) according to the manufacturer's instructions. Briefly, cells were plated 24 hours before transfection at a density of $3 \times 10^{5} /$ well on a 12 well tissue culture dish (Corning Costar). The next day cells were transfected with $1.5 \mu \mathrm{g}$ of Egr-1 reporter gene DNA (kindly provided by Dr William Aird, Beth Israel Deaconess Medical Center, Boston, Massachusetts, USA). Thirty hours post transfection, media was removed and cells were incubated with serum free medium for 16 hours to reduce background prior to stimulation with $H$ pylori or PMA (positive control) for four hours. Cells were then lysed and reporter activity measured in each sample using a luciferase reporter assay system (Promega) according to the manufacturer's instructions. In some experiments, a plasmid construct encoding a dominant negative MEKl (kindly provided by Dr Sang Hoon Rhee, Beth Israel Deaconess Medical Center) or vector alone was cotransfected with the Egr-1 construct. All assays were performed in triplicate and a single representative experiment is shown. Data are expressed as mean (SEM).

\section{Statistical analyses}

Statistical analyses were performed using Sigma-Stat for Windows version 2.0 (Jandel Scientific Software, San Rafael, California, USA). Analysis of variance followed by protected 


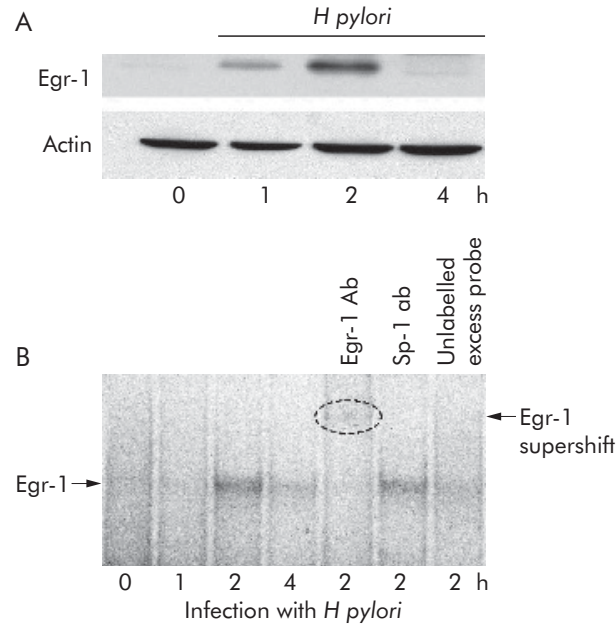

Figure 1 Early growth response gene 1 (Egr-1) is upregulated in Helicobacter pylori infected gastric epithelial cells. (A) Quiescent AGS cells were treated with $H$ pylori cag+ ATCC strain 43504 (MOI 20:1) for the indicated times. Equal amounts of cell extracts were used to determine levels of Egr-1, as described in the materials and methods section. The upper panel shows Egr-1 levels present in cell lysates, the lower panel actin, which acts as a control for loading. The results are representative of three independent experiments. (B) Serum starved $100 \mathrm{~mm}$ plates of confluent AGS cells infected with $\mathrm{H}$ pylori cag+ ATCC strain 43504 (MOI 20:1) over a four hour time course. Nuclear extracts were made and electrophoretic mobility gel shifts were performed using a radiolabelled Egr-1 consensus sequence, as described in the materials and methods section. A supershift analysis to confirm the complex contained Egr-1 was also performed. Nuclear extracts from AGS cells infected with $H$ pylori for two hours were incubated with excess cold probe or antibodies (ab) against Egr-1 and Sp-1 and then subjected to electrophoretic mobility shift assay. Supershift with Egr-1 antibody can be seen in the fifth lane, as indicated by the arrow.

$t$ tests were used for intergroup comparisons, except where otherwise stated.

\section{RESULTS}

\section{Egr- 1 is upregulated in $H$ pylori infected gastric epithelial cells}

Egr- 1 is an immediate early gene and transcription factor that can be rapidly upregulated by numerous stimuli. Increased levels of Egr-1 can lead to changes in a number of biological processes, such as proliferation, inflammation, and cancer progression. ${ }^{18}$ To determine whether $H$ pylori (cag+ ATCC strain 43504) could upregulate Egr-1, we infected AGS cells over a four hour time course with the bacteria and then examined whole cell lysates with an Egr-l antibody. As shown in fig IA, $H$ pylori upregulated Egr-l after one hour, this was maximal at two hours, and levels returned to baseline by four hours. Identical results to those seen with AGS cells were also seen with another gastric epithelial cell line, MKN-28 (data not shown).

To demonstrate that the Egr-l upregulated in $H$ pylori infected gastric epithelial cells is capable of binding to Egr-l target genes, we isolated nuclear extracts from cells infected with $H$ pylori over the four hour time course and performed an EMSA using an Egr consensus sequence. As shown in fig 1B, nuclear extracts from untreated cells showed little Egr-l binding. After infection with $H$ pylori for two hours, maximal binding occurred which was again reduced by four hours. To determine whether Egr-1 was binding to the probe, a supershift experiment was also performed. Nuclear extracts prepared from AGS cells were incubated with either an antibody to Egr-1 or Sp-1 as a control. The complex only underwent a supershift in the presence of the Egr-1 antibody (fig 1B, 5th lane, supershift indicated by arrow); in contrast
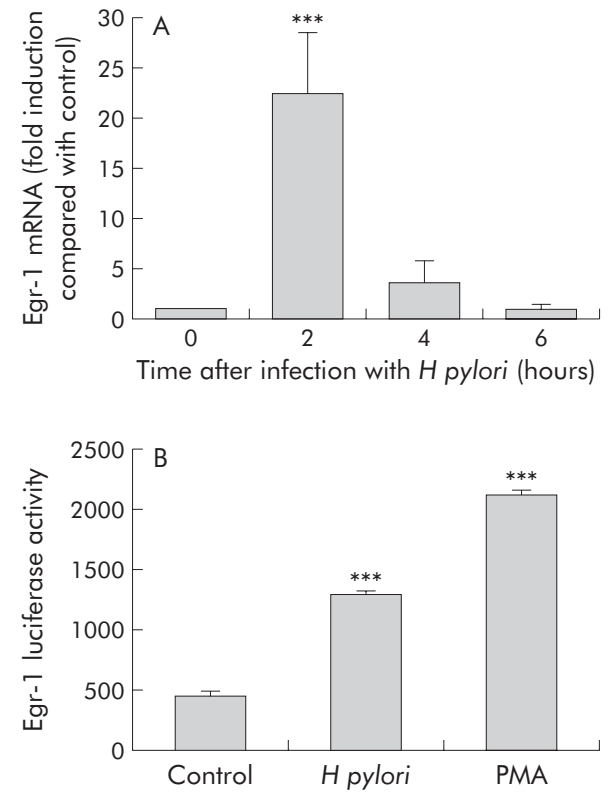

Figure 2 Helicobacter pylori infection results in increased early growth response gene 1 (Egr-1) mRNA expression. (A) Infection of AGS cells with $H$ pylori resulted in an increase in Egr-1 mRNA expression over a six hour time course, as determined by real time polymerase chain reaction. Data were normalised to glyceraldehyde-3-phosphate dehydrogenase levels and results are expressed as fold induction above the unstimulated control. (B) H pylori infection upregulated Egr-1 luciferase reporter activity. Cells were transfected for 48 hours with an Egr-1 luciferase reporter construct. Cells were then treated with media alone (control), H pylori cag+ ATCC strain 43504 (MOI 20:1), or phorbol-12-myristate-13-acetate (PMA $10 \mathrm{nM}$ ) for four hours. Luciferase activity was then measured in cell lysates. Values are expressed as mean (SEM); $n=3$ ). ${ }^{* * *} p<0.001$ versus unstimulated control.

no supershift was observed with the Sp-1 antibody. These results confirm that Egr-l was present in the complex binding to our Egr consensus sequence.

\section{$H$ pylori infection results in increased Egr- 1 mRNA expression and increases the activity of an Egr-1 luciferase reporter construct}

We next examined whether infection of AGS cell with cag $+H$ pylori could increase Egr-1 mRNA expression. Analysis of CDNA by real time PCR revealed a 22 -fold increase in Egr-1 mRNA levels at the two hour time point, which returned to baseline levels by six hours (fig 2A). These findings

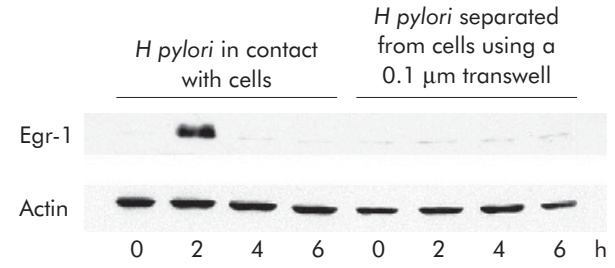

Figure 3 Early growth response gene 1 (Egr-1) upregulation is mediated through contact between the bacterium and the cell. Serum starved AGS cells were exposed directly to Helicobacter pylori or cocultured with $H$ pylori separated by a $0.1 \mu \mathrm{m}$ filter that prevented direct contact between the bacteria and epithelial cells. The experiment was performed over a six hour time course and Egr-1 levels were analysed, as described in the materials and methods section. The upper panel shows Egr-1, the lower panel actin, used as a loading control. The results are representative of three independent experiments. 
demonstrate that infection of AGS cells with $H$ pylori results in a rapid but transient increase in Egr-l mRNA levels, consistent with the Egr-1 protein data presented in fig 1 . We examined whether infecting AGS cells with $H$ pylori could induce activation of an Egr-1 luciferase reporter construct. AGS cells transfected with an Egr-1 reporter construct were treated with either $H$ pylori or our positive control (PMA) for four hours and luciferase activity of the cells was then measured. Infecting AGS cells with $H$ pylori resulted in a 2.9fold increase in reporter activity while treatment with PMA resulted in a 4.7-fold increase above control (fig $2 \mathrm{~B}$ ).

\section{Egr-1 upregulation is mediated through contact between the bacterium and the cell}

Next we tested whether soluble factors produced by the bacterium played a role in Egr-l upregulation or whether contact between bacterium and cell was necessary for induction using a Transwell system. We found that by preventing direct contact between AGS cells and the bacteria, upregulation of Egr-1 was completely prevented, suggesting that contact, and not soluble factors, plays a role in this process (fig 3).

\section{Egr- 1 is differentially upregulated by cag+ and cag- strains of $\boldsymbol{H}$ pylori}

Our group and others have previously shown that cag+ and cag - strains of $H$ pylori are capable of differentially activating signal transduction pathways in gastric epithelial cells. ${ }^{4-6}$ To determine whether Egr-1 was also preferentially upregulated by cag+ versus cag- strains of $H$ pylori, we infected AGS gastric epithelial cells with a panel of three cag+ and 3 cagstrains of $H$ pylori for two hours. Whole cell lysates were then analysed by western blotting using an Egr-l specific antibody. As shown in fig 4A, cag + strains of $H$ pylori were capable of inducing Egr-1 production at two hours but in AGS cells infected with cag- strains, this induction did not occur. In addition, we also examined upregulation of Egr-l in AGS cells infected with each of the six strains over a six hour time course. Figure 4B shows a complete six hour time course for

A

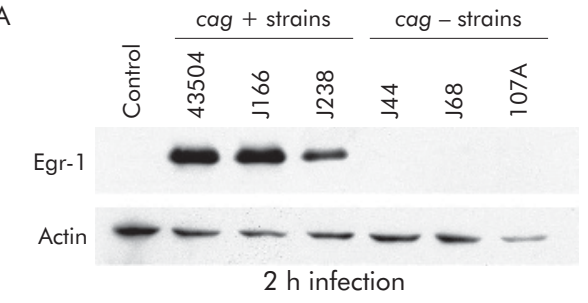

B

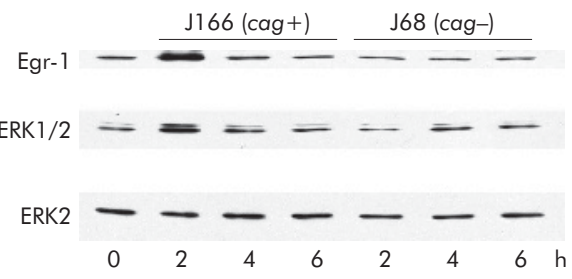

Figure 4 Early growth response gene 1 (Egr-1) is differentially upregulated by cag+ and cag- strains of Helicobacter pylori. (A) Serum starved AGS cells were treated with a panel of three cag+ or three cagstrains of $H$ pylori for two hours. Levels of Egr-1 protein present in the cell lysates were determined by western blotting, as described in the materials and methods section. The upper panel shows Egr-1 levels present in cell lysates, the lower panel shows levels of actin, used as a loading control. Results are representative of three independent experiments. (B) Quiescent AGS cells were infected with a cag+ (J166) or cag- (J68) strain of $\mathrm{H}$ pylori over a six hour time course. Lysates were analysed for Egr-1, phosphorylated extracellular regulated kinase (ERK) $1 / 2$, and ERK2 by western blotting. the J166 cagt strain and the J68 cag- strain, again demonstrating an increase in Egr-1 levels (3.2-fold) at two hours occurring with infection with the cag+ strain, but not with the cag - strain. As Egr-1 upregulation is known to be regulated via activation of the ERKI/2 signalling pathway, ${ }^{23}$ we also analysed the blots for increases in ERK1/2 phosphorylation. Our findings (fig 4B) showed that increased ERKl/2 phosphorylation (2.4-fold at two hours) appeared to correlate with the increases seen in Egr-1 levels.

\section{Egr- 1 upregulation by $H$ pylori is dependent on an intact cag secretion system, but not CagA}

Previously, we and other investigators have shown that the cag pathogenicity island of $H$ pylori plays an important role in activating various signal transduction pathways within gastric epithelial cells. ${ }^{4-6}$ To determine the importance of the cag secretion system to upregulation of Egr- 1 by $H$ pylori, we compared an isogenic cagE- mutant of $H$ pylori with its parental strain. The cagE gene has previously been found to be essential to the functioning of the type IV secretion system. For this experiment we infected AGS cells with wildtype H pylori strain J166 or its cagE - isogenic mutant over a six hour time course. Lysates were then collected and analysed by western blotting to determine the level of Egr-1 present in the cells. As shown in fig 5A, the cagE- mutant had an impaired ability to upregulate Egr-1 protein levels compared with the parent strain. Analysis of Egr-1 mRNA levels by real time PCR also revealed that the cagE - mutant was much less efficient in upregulating Egr-1 mRNA levels ( $\sim 10$ fold less after two hours of infection) than its wild-type counterpart. ERKl/2 phosphorylation was also upregulated by the wild-type strain, but not the cagE- mutant at the two hour time point, again correlating with the increase in Egr-1 levels. These findings were confirmed using cagE - isogenic mutant derived from parental strain 60190 (data not shown).

We next examined whether CagA participated in $H$ pylori mediated Egr-1 upregulation. Cells were incubated with wild-type $H$ pylori strain 60190 or a cagA- isogenic mutant
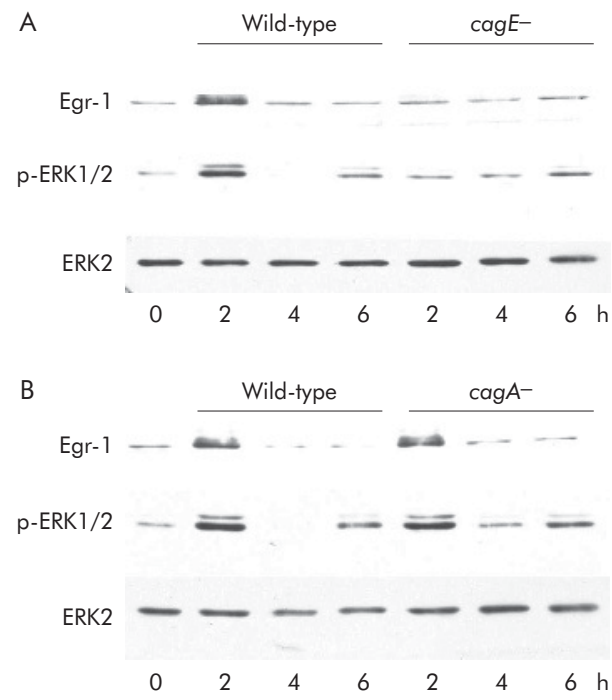

Figure 5 Early growth response gene 1 (Egr-1) upregulation by Helicobacter pylori is dependent on an intact cag pathogenicity island. (A, B) Serum starved AGS cells were treated with a $\mathrm{H}$ pylori cag+ strain $\mathrm{J} 166$ or its isogenic cagE - or cagA- mutants, over a six hour time course. Western blotting was used to determine levels of Egr-1 present in the cell lysates, as described in the materials and methods section. Upper panel shows Egr-1 levels present in cell lysates, the middle panel phosphorylated extracellular regulated kinase (ERK) $1 / 2$, and the bottom panel ERK2, which acted as a control for loading. 
over a six hour time course and their ability to upregulate Egr-1 protein was examined. As shown in fig 5B, the cagAmutant, unlike the cagE - mutant, had a similar ability to upregulate Egr-1 levels compared with the parental strain. These findings indicate that induction of Egr- 1 in AGS cells, although requiring the participation of the type IV secretion system, is not dependent on translocation of CagA. Again, ERK1/2 phosphorylation was examined, and appeared to correlate with increases in Egr-l levels. These results were also confirmed using our second set of isogenic mutants derived from cag+ strain 60190 (data not shown).

\section{ERK 1 / 2 activation is required for Egr- 1 upregulation} in $\boldsymbol{H}$ pylori infected gastric epithelial cells

We and other investigators have previously reported that $H$ pylori can activate numerous signal transduction pathways, including the ERK1/2, p38, and c-Jun N-terminal kinase (JNK) MAP kinase pathways. ${ }^{4-6} 24$ Therefore, we investigated whether MAP kinase signalling pathways were involved in Egr-1 upregulation. AGS cells were treated with a number of pharmacological inhibitors, including the ERKI/2 inhibitor PD98059 $(25 \mu \mathrm{M})$, p38 inhibitor SB203580 $(10 \mu \mathrm{M})$, and the JNK inhibitor SP600125 (50 $\mu \mathrm{M})$. Only the ERKl/2 inhibitor significantly decreased (69\%) Egr-1 protein levels (fig 6A). Inhibitors of p38 and JNK were unable to prevent Egr-1 upregulation in $H$ pylori infected cells (data not shown). These findings indicate that ERK1/2 phosphorylation in part is required for $H$ pylori mediated Egr-l upregulation in AGS cells. In order to confirm our pharmacological data that ERKl/2 was involved in this process, we utilised a dominant negative MEKl construct, which was cotransfected into our
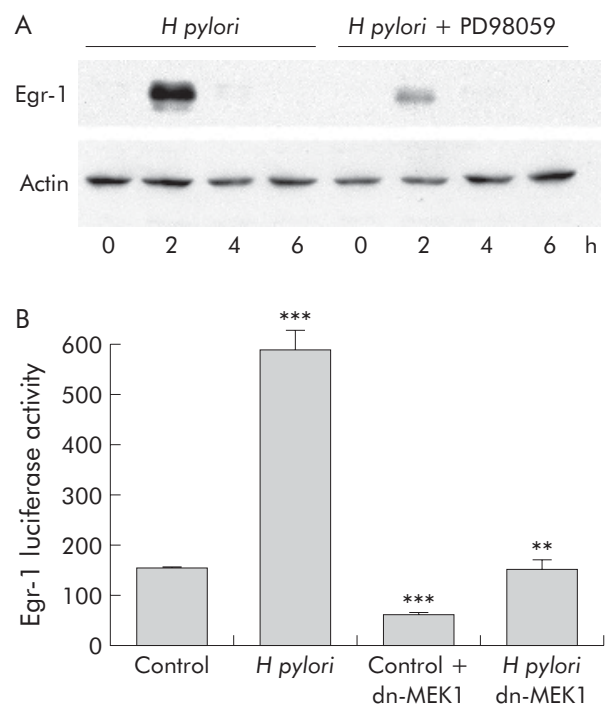

Figure 6 The extracellular regulated kinase (ERK) $1 / 2$ signalling pathway is upstream of early growth response gene 1 (Egr-1) upregulation in Helicobacter pylori infected gastric epithelial cells. (A) Quiescent AGS cells were pretreated with $25 \mu \mathrm{M}$ of the MEK1 inhibitor PD98059 for 30 minutes and then cotreated with $\mathrm{H}$ pylori cag+ ATCC strain 43504 (MOI 20:1) over a six hour time course. Western blotting was used to determine the levels of Egr-1 present in the cell lysates, as described in the materials and methods section. The upper panel shows Egr-1 levels present in cell lysates, the lower panel actin, used as a control for loading. Results are representative of three independent experiments. (B) Cells were cotransfected with an Egr-1 luciferase reporter and a dominant negative (dn) MEKI overexpression construct or control DNA. Cells were then treated with media alone (control) or H pylori cag+ ATCC strain 43504 (MOI 20:1) for four hours. Luciferase activity was then measured in cell lysates. Values are expressed as mean (SEM); $n=3$ ). ${ }^{* * *} \mathrm{p}<0.001$, control versus control + dn-MEK $1 ;{ }^{* *} \mathrm{p}<0.01, H$ pylori versus $H$ pylori + dn-MEK 1 .

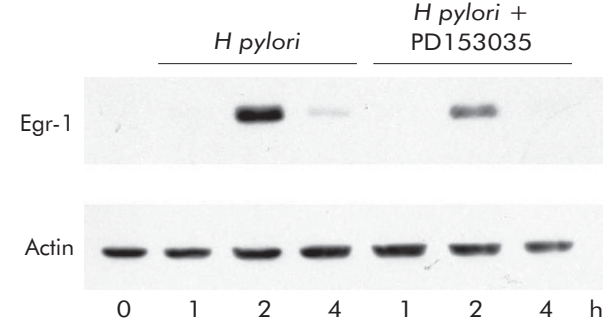

Figure 7 Epidermal growth factor receptor (EGFR) transactivation plays a role in Helicobacter pylori induced early growth response gene 1 (Egr-1) upregulation. AGS cells were pretreated with the EGFR kinase inhibitor PD $153035(5 \mu \mathrm{M})$ for 30 minutes and then infected with $H$ pylori over a four hour time course. Egr-1 protein levels were analysed by western blotting, as described in the materials and methods section. Top panel shows Egr-1 levels, the lower panel actin, used as a control for loading. Results are representative of three independent experiments.

cells along with our Egr-1 luciferase reporter construct. As shown in fig 6B, overexpression of dominant negative MEKl resulted in a $40 \%$ and $75 \%$ reduction in basal and $H$ pylori stimulated Egr-1 luciferase reporter activity, respectively, compared with cells cotransfected with the Egr-l reporter and vector control.

\section{Egr- 1 upregulation in $H$ pylori can be downregulated by blocking epidermal growth factor receptor (EGFR) transactivation}

We have previously identified EGFR transactivation as one mechanism leading to the regulation of ERKl/2 phosphorylation by $H$ pylori. ${ }^{20}$ We therefore examined whether blockade of EGFR kinase activity could similarly reduce $H$ pylori stimulated Egr-1 protein levels. Cells were pretreated with the EGFR kinase PD153035 $(5 \mu \mathrm{M})$ for 30 minutes and then infected with $H$ pylori over a four hour time course. Egr- 1 protein levels were then examined by western blotting. As shown in fig 7, EGFR kinase inhibitor treatment resulted in partial blockade ( $\sim 50 \%$ ) of H pylori mediated Egr-1 upregulation. Similar data were also obtained with the EGFR kinase inhibitor AG1478 ( $1 \mu \mathrm{M})$. These findings indicate that EGFR transactivation plays a role in $H$ pylori mediated Egr-l upregulation in AGS gastric epithelial cells.

\section{DISCUSSION}

Previous studies have shown that infection with $H$ pylori is associated with increased gastric epithelial cell proliferation. ${ }^{16}$ Increased cell turnover is an important risk factor for increased mutations and the progression to cancer. Egr-l is a transcription factor that is able to regulate numerous genes involved in the proliferative response, which include metalloproteinases, growth factors, and their receptors. ${ }^{18}$ Therefore, Egr-1 upregulation may be an important event in $H$ pylori associated cancer progression.

In this study, we showed that infection of gastric epithelial cells with $H$ pylori resulted in a rapid but transient increase in Egr-1, both at the mRNA and protein levels. Although upregulation of Egr- 1 in gastric epithelial cells by $H$ pylori has previously been described by one other group, ${ }^{25}$ our study extends these observations in several important ways. We showed differences in induction of Egr-l between cag+ and cag- $H$ pylori, demonstrated the requirement for an intact PAI, and showed that $H$ pylori mediated EGFR transactivation and ERK1/2 activation are upstream of Egr-1 upregulation.

One of the more significant findings of this study is that cag+ strains of $H$ pylori are more potent in their ability to upregulate Egr-1 than cag- strains Furthermore, $H$ pylori that have a non-functioning type IV secretion system due to mutation of the cagE gene also produce weaker activation of 
Egr-1. In previous studies it has been shown that infection of gastric epithelial cells with either $H$ pylori cag+ and cagstrains results in divergent responses. Signal transduction pathways such as the MAP kinase cascades, activation of transcription factors such as nuclear factor $\kappa \mathrm{B}$, and upregulation of other early response genes such as c-fos are all preferentially induced by cag+ strains. ${ }^{458}$ Infection with cag+ strains similarly results in upregulation of genes involved in the immune response, cell turnover, and apoptosis. This may explain why individuals infected with cag+ strains are more likely to develop atrophic gastritis and distal gastric cancer than those infected with cag- strains. ${ }^{14}$

We and others have shown that $H$ pylori are capable of causing transactivation of the EGFR via metalloproteinase dependent cleavage of HB-EGF. ${ }^{20}{ }^{26}$ We have previously found that cag- strains of $H$ pylori are less able to induce transactivation of the EGFR than their cag+ counterparts. ${ }^{20}$ Likewise, mutants with disrupted type IV secretion systems are less able than their parental strains to induce EGFR transactivation. This in turn affects the ability of bacteria to induce early ERKl/2 phosphorylation, an event we have determined is necessary for Egr-1 upregulation. It has been found that ERK1/2 phosphorylation can also be mediated by soluble secreted factors ${ }^{27}$ that are independent of the cag PAI. Our Transwell experiments suggest that these soluble factors do not participate in the rapid Egr-1 upregulation observed in our model system.

Infection of our cells with $\operatorname{cag} A-$ mutants resulted in a similar pattern of Egr-1 induction to that of the wild-type strain at early time points. However, later time points ( $>6$ hours) were not examined in this study. Several groups have reported that CagA interacts with numerous host proteins, including Grb2, which results in sustained activation of the ERKl/2 MAP kinase pathway up to 24 hours. ${ }^{28} 29$ This may potentially lead to Egr-1 upregulation and thus we cannot rule out the possibility that CagA may have effects on Egr-1 upregulation at later stages.

In our in vitro model $H$ pylori induced Egr-l upregulation appeared to be short lived. This is a typical activation pattern seen with other stimuli, including growth factors, hormones, and neurotransmitters. ${ }^{18}$ Egr- 1 gene regulation is tightly controlled by two transcriptional cofactors termed NFGFI-A binding proteins 1 and 2 (NAB1, NAB2). ${ }^{18}$ It has been demonstrated that NAB2 gene expression is itself regulated by Egr- 1 and therefore Egr- 1 controls its own activity through a negative feedback loop. ${ }^{30}$ Interestingly, Egr-1 mRNA expression and protein production have been found to be significantly higher in gastric cancer tissues than in normal mucosa. ${ }^{19}$ We speculate that since $H$ pylori chronically infects the stomach, sometimes for many decades, constant reexposure of the epithelium to the bacterium may cause frequent cellular activation and upregulation of Egr-1. Moreover, Egr-l has also been identified as a key transcriptional regulator of EGFR ${ }^{31}$ and other growth factors and their receptors. $^{32}$ Thus its continual activation over time may contribute to gastric epithelial cell hyperproliferation, a known risk factor for gastric carcinogenesis.

In addition to being upregulated in gastric cancer, Egr-1 has been found to play a particularly important role in prostate and breast cancer. ${ }^{33}{ }^{34}$ Egr-1 expression levels are elevated in human prostate carcinomas in proportion to grade and stage, and in two models of prostate cancer, prostate cancer progression was found to be significantly delayed in mice lacking Egr-1. ${ }^{34}$ In a recent publication by Mitchell et al, DNAzymes targeting Egr-l inhibited human breast carcinoma proliferation, migration, chemoinvasion, and solid tumour growth. ${ }^{33}$ Collectively, these findings together with the data presented in this study suggest that
Egr-1 may play an important role in $H$ pylori mediated carcinogenesis.

\section{ACKNOWLEDGEMENTS}

This work was supported by NIH grants DK54920 and DK58858 (CP Kelly) and NIH grants CA 77955 and DK 58587 (RM Peek Jr). AC Keates is a recipient of a FIRST Award from the Crohn's and Colitis Foundation of America.

\section{Authors' affiliations}

S Keates, A C Keates, S Nath, C P Kelly, Division of Gastroenterology, Beth Israel Deaconess Medical Center, Harvard Medical School, Boston, MA, USA

R M Peek Jr, Division of Gastroenterology, Vanderbilt University Medical Center, Nashville, TN, USA

Conflict of interest: None declared.

\section{REFERENCES}

1 Kuipers EJ, Thijs JC, Festen HP. The prevalence of Helicobacter pylori in peptic ulcer disease. Aliment Pharmacol Ther 1995;9(suppl 2):59-69.

2 Wotherspoon AC, Ortiz-Hidalgo C, Falzon MR, et al. Helicobacter pyloriassociated gastritis and primary B-cell gastric lymphoma. Lancet 1991;338:1175-6.

3 Huang JQ, Sridhar S, Chen Y, et al. Meta-analysis of the relationship between Helicobacter pylori seropositivity and gastric cancer. Gastroenterology 1998; 114:1169-79.

4 Keates S, Keates AC, Warny M, et al. Differential activation of mitogenactivated protein kinases in AGS gastric epithelial cells by cag+ and cagHelicobacter pylori. J Immunol 1999; 163:5552-9.

5 Meyer-ter-Vehn T, Covacci A, Kist M, et al. Helicobacter pylori activates mitogen-activated protein kinase cascades and induces expression of the proto-oncogenes c-fos and c-jun. J Biol Chem 2000;275:16064-72.

6 Naumann M, Wessler S, Bartsch C, et al. Activation of activator protein 1 and stress response kinases in epithelial cells colonized by Helicobacter pylori encoding the cag pathogenicity island. J Biol Chem 1999;274:31655-62

7 Keates S, Hitti YS, Upton M, et al. Helicobacter pylori infection activates NFkappa B in gastric epithelial cells. Gastroenterology 1997;113:1099-109.

8 Glocker E, Lange C, Covacci A, et al. Proteins encoded by the cag pathogenicity island of Helicobacter pylori are required for NF-kappaB activation. Infect Immun 1998;66:2346-8.

9 Aihara M, Tsuchimoto D, Takizawa $\mathrm{H}$, et al. Mechanisms involved in Helicobacter pylori-induced interleukin-8 production by a gastric cancer cell line, MKN45. Infect Immun 1997;65:3218-24.

10 Censini S, Lange C, Xiang Z, et al. cag, a pathogenicity island of Helicobacter pylori, encodes type I-specific and disease-associated virulence factors. Proc Natl Acad Sci U S A 1996;93:14648-53.

11 Asahi M, Azuma T, Ito S, et al. Helicobacter pylori CagA protein can be tyrosine phosphorylated in gastric epithelial cells. J Exp Med 2000;191:593-602.

12 Odenbreit S, Puls J, Sedlmaier B, et al. Translocation of Helicobacter pylori CagA into gastric epithelial cells by type IV secretion. Science 2000;287: 1497-500.

13 Stein M, Rappuoli R, Covacci A. Tyrosine phosphorylation of the Helicobacter pylori CagA antigen after cag-driven host cell translocation. Proc Natl Acad Sci U S A 2000;97:1263-8.

14 Peek RM Jr. Helicobacter pylori strain-specific modulation of gastric mucosal cellular turnover: implications for carcinogenesis. J Gastroenterol 2002;37(suppl 13):10-16.

15 Viala J, Chaput C, Boneca IG, et al. Nod1 responds to peptidoglycan delivered by the Helicobacter pylori cag pathogenicity island. Nat Immunol 2004;5: $1166-74$

16 Peek RM Jr, Moss SF, Tham KT, et al. Helicobacter pylori cagA+ strains and dissociation of gastric epithelial cell proliferation from apoptosis. J Natl Cancer Inst 1997; 89:863-8.

17 Rokkas T, Ladas S, Liatsos C, et al. Relationship of Helicobacter pylori CagA status to gastric cell proliferation and apoptosis. Dig Dis Sci 1999;44:487-93.

18 Thiel G, Cibelli G. Regulation of life and death by the zinc finger transcription factor Egr-1. J Cell Physiol 2002;193:287-92.

19 Kobayashi D, Yamada M, Kamagata C, et al. Overexpression of early growth response-1 as a metastasis-regulatory factor in gastric cancer. Anticancer Res 2002;22:3963-70.

20 Keates S, Sougioultzis S, Keates AC, et al. cag+ Helicobacter pylori induce transactivation of the epidermal growth factor receptor in AGS gastric epithelial cells. J Biol Chem 2001;276:48127-34.

21 Tummuru MK, Sharma SA, Blaser MJ. Helicobacter pylori picB, a homologue of the Bordetella pertussis toxin secretion protein, is required for induction of IL-8 in gastric epithelial cells. Mol Microbiol 1995;18:867-76.

22 Keates S, Keates AC, Mizoguchi E, et al. Enterocytes are the primary source of the chemokine ENA-78 in normal colon and ulcerative colitis. Am J Physiol 1997;273:G75-82.

23 Al-Sarraj A, Thiel G. Substance P induced biosynthesis of the zinc finger transcription factor Egr-1 in human glioma cells requires activation of the epidermal growth factor receptor and of extracellular signal-regulated protein kinase. Neurosci Lett 2002;332:111-14. 
24 Crawford HC, Krishna US, Israel DA, et al. Helicobacter pylori strain-selective induction of matrix metalloproteinase-7 in vitro and within gastric mucosa. Gastroenterology 2003;125:1125-36

25 Abdel-Latif MM, Windle HJ, Fitzgerald KA, et al. Helicobacter pylori activates the early growth response 1 protein in gastric epithelial cells. Infect Immun 2004:72:3549-60

26 Wallasch C, Crabtree JE, Bevec D, et al. Helicobacter pylori-stimulated EGF receptor transactivation requires metalloprotease cleavage of HB-EGF. Biochem Biophys Res Commun 2002;295:695-701.

27 Wessler S, Rapp UR, Wiedenmann B, et al. B-Raf/Rapl signaling, but not CRaf-1/Ras, induces the histidine decarboxylase promoter in Helicobacter pylori infection. Faseb J 2002;16:417-19.

28 Mimuro H, Suzuki T, Tanaka J, et al. Grb2 is a key mediator of Helicobacter pylori CagA protein activities. Mol Cell 2002; 10:745-55

29 Higashi H, Nakaya A, Tsutsumi R, et al. Helicobacter pylori CagA induces Ras-independent morphogenetic response through SHP-2 recruitment and activation. J Biol Chem 2004;279:17205-16.
30 Ehrengruber MU, Muhlebach SG, Sohrman S, et al. Modulation of early growth response (EGR) transcription factor-dependent gene expression by using recombinant adenovirus. Gene 2000;258:63-9

31 Nishi $\mathrm{H}$, Nishi KH, Johnson AC. Early growth response-1 gene mediates upregulation of epidermal growth factor receptor expression during hypoxia. Cancer Res 2002;62:827-34.

32 Khachigian LM, Williams AJ, Collins T. Interplay of Spl and Egr-1 in the proximal platelet-derived growth factor A-chain promoter in cultured vascular endothelial cells. J Biol Chem 1995:270:27679-86.

33 Mitchell A, Dass CR, Sun $L Q$, et al. Inhibition of human breast carcinoma proliferation, migration, chemoinvasion and solid tumour growth by DNAzymes targeting the zinc finger transcription factor EGR-1. Nucleic Acids Res 2004;32:3065-9.

34 Baron V, De Gregorio G, Krones-Herzig A, et al. Inhibition of Egr-1 expression reverses transformation of prostate cancer cells in vitro and in vivo. Oncogene 2003;22:4194-204.

\section{EDITOR'S QUIZ: GI SNAPSHOT}

\section{Classical signs: modern imaging}

\section{Clinical presentation}

A 64 year old man presented with a two month history of increasing dysphagia and weight loss. Upper gastrointestinal endoscopy was performed and a lesion was identified in the

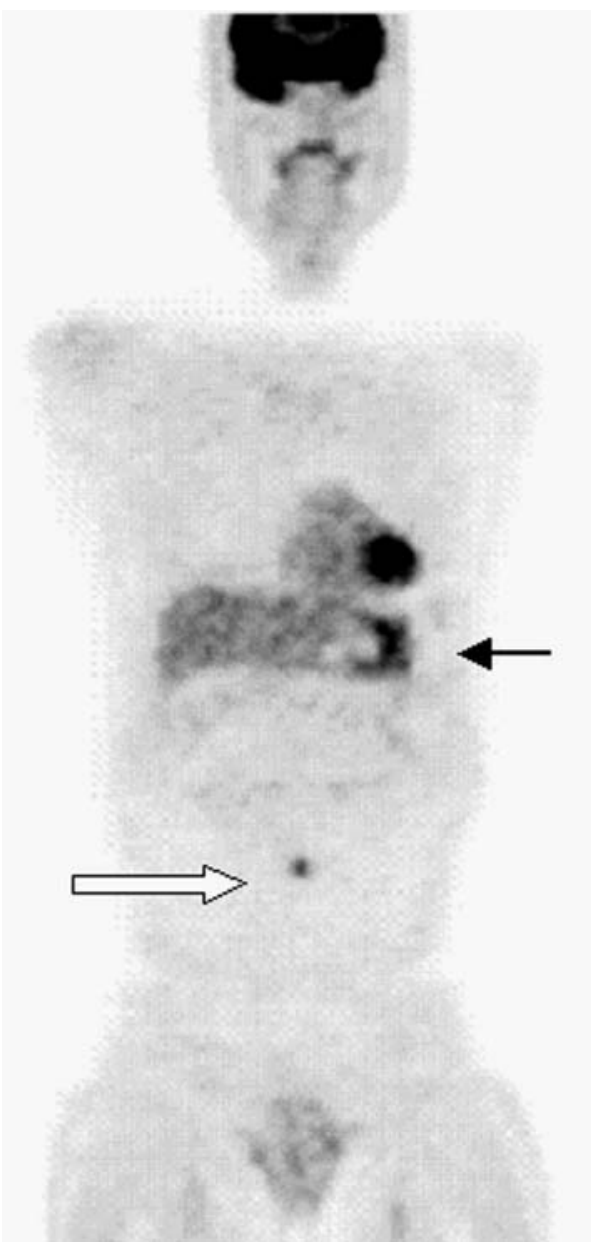

Figure 1 An anterior coronal image from a half body ${ }^{18} \mathrm{~F}-$ fluorodeoxyglucose positron emission tomography examination.

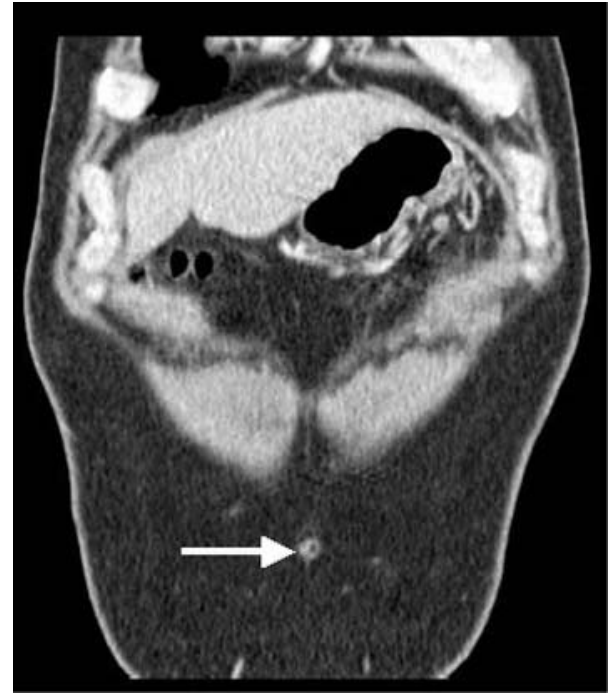

Figure 2 Coronal reconstruction from a 16 detector multislice computed tomography image of the anterior abdomen.

region of the gastro-oesophageal junction, which was biopsied. The patient then underwent both a ${ }^{18} \mathrm{~F}$-fluorodeoxyglucose positron emission tomography study (fig l) and a 16 detector computed tomography examination (fig 2).

\section{Question}

What abnormality is the white arrow pointing to on both images?

See page 1395 for answer

This case is submitted by:

A M Groves

Department of Radiology, Addenbrooke's Hospital, Cambridge, UK

K K Balan

Department of Nuclear Medicine, Addenbrooke's Hospital, Cambridge, UK

Correspondence to: Dr A M Groves, Radiology Department, Box 219 , Addenbrooke's Hospital, Hills Rd, Cambridge, CB2 2QQ, UK; drashleygroves@hotmail.com

doi: $10.1136 /$ gut.2005.068338 